\title{
ON A SERIES OF GOLDBACH AND EULER
}

\author{
PELEGRÍ VIADER, LLUÍS BIBILONI, AND JAUME PARADÍS
}

\begin{abstract}
Theorem 1 of Euler's paper of 1737 "Variae Observationes Circa Series Infinitas," states the astonishing result that the series of all unit fractions whose denominators are perfect powers of integers minus unity has sum one. Euler attributes the Theorem to Goldbach. The proof is one of those examples of misuse of divergent series to obtain correct results so frequent during the seventeenth and eighteenth centuries. We examine this proof closely and, with the help of some insight provided by a modern (and completely different) proof of the Goldbach-Euler Theorem, we present a rational reconstruction in terms which could be considered rigorous by modern Weierstrassian standards. At the same time, with a few ideas borrowed from nonstandard analysis we see how the same reconstruction can be also be considered rigorous by modern Robinsonian standards. This last approach, though, is completely in tune with Goldbach and Euler's proof. We hope to convince the reader then how, a few simple ideas from nonstandard analysis, vindicate Euler's work.
\end{abstract}

Key words. History of mathematics, infinite series, nonstandard analysis.

\section{Journal of Economic Literature Classification: C00}

Mathematics Subject Classifications (1991). 01A50; 26E35.

\section{INTRODUCTION}

Euler's paper Variae Observationes Circa Series Infinitas, [7], ought to be considered important for several reasons. It contains the first printed version of Euler's product for Riemann's zeta function; it definitely establishes the use of the symbol $\pi$ to denote the perimeter of the circle of diameter one, and it introduces a legion of interesting infinite products and series. The first of these is Theorem 1 which Euler says was communicated and proved to him by Goldbach in a letter (now lost):

$$
\sum_{m, n \geq 2} \frac{1}{m^{n}-1}=1
$$

(One must avoid repetitions in the above sum). We will refer to this result as the Goldbach-Euler Theorem (GET).

Date: June 2004. 
Goldbach and Euler's proof is a typical example of what some historians consider a misuse of divergent series as it starts assigning a "value" to the harmonic series $\sum 1 / n$ and proceeds by manipulating it by substraction and replacement of other series till reaching the desired result. This unchecked use of divergent series to obtain valid results was a standard procedure in the late seventeenth and early eighteenth centuries. It has raised quite a lot of criticism, corrections and, why not, praise caused by the audacity of the mathematicians of the time, led by Euler, the Master of us All, as Laplace baptized him. We offer Goldbach and Euler's proof in section 2. We also make some considerations about the use of divergent series as a method of proof examining another famous instance of exactly the same kind of manipulations: the 'proof' of the divergence of the harmonic series by Jakob Bernoulli. In this last case, though, the conclusion was a contradiction instead of a valid result. As it happens, the same procedure led to different answers! Despite Euler being completely aware of this apparent paradox he did not question Goldbach's proof of the GET at all. That means that his confidence in the use of the harmonic series and its infinite sum was based on something more solid than sheer audacity.

There are quite a number of papers devoted, either directly or indirectly, to Euler's use of the infinitely large and the infinitely small. A recent one by Detlef Laugwitz is specially relevant [12]. Part I of this paper bears the title "The algorithmic thinking of Leibniz and Euler". The word 'algorithmic' in the title emphasizes the point of view that Leibniz and Euler used 'infinitely large' and 'infinitely small' numbers as any other real number. Laugwitz argues that Euler was "more interested in algorithmic applications" than in "conceptual arguments" without being altogether careless for, in some cases, it is possible to verify his results "rigorously" by the proper limit considerations. In Laugwitz words,

It is not difficult to verify 'rigourously' these sums of series by considering finite partial sums and passing to the limit. But for Euler there were no limit considerations, and what we want to know is the kind of reasoning employed by him and by his contemporaries. This being so it would be counterproductive to handle these problems using perfected later means. [12, p. 450] [Our emphasis]

Thus, at the base of Euler's reasoning there must have been something consistent enough to make most of his results correct. These apparently solid foundations come from a concept of number sufficiently enlarged so as to encompass the infinitely small and the infinitely large harmonically. Our modern model for that is what is called nonstandard analysis after Abraham Robinson's monograph [16]. We give a very 
short account of the basic ideas behind nonstandard analysis in section 3. But even these few concepts will suffice to review the proof of the GET vindicating in this way Goldbach and Euler's work.

As a preliminary test for the power of nonstandard tools, section 4 reconstructs Jakob Bernoulli's proof. We see how, either using perfected later means or through a nonstandard prism the same wording shows that the contradiction is, in fact, a tautology!

A similar reconstruction can be done with the proof of the GET. In order to do so, we use some ideas drawn from a modern (and completely different) proof of the GET which appeared in this MonTHLY [15] as a solution to a previously proposed problem [17]. This proof, reproduced in section 5 , is extremely short but very appealing. In the same section we examine in detail the line of thought behind it. This will not only be an interesting exercise on its own but will also reveal some unexpected and very simple results to be used later on. As a more recent reference let us mention that a different, new - though much longer - proof of the GET appeared in [1].

We devote section 6 to the reconstruction of Goldbach and Euler's proof. We re-read it both from a passage-to-the-avoiding point of view and from the nonstandard point of view. We show how the same arguments used by Euler, slightly modified, become rigorous for our modern standards. And, as in the case of Bernoulli's proof, the main point is that almost exactly the same wording can be considered a Weierstrassian proof or a nonstandard one.

In the epilog we show that in the same Variae Observationes other results proved using the same techniques are not so easily amended as the GET and how, in some instances, some blunders make their way into Euler's otherwise brilliant analysis.

\section{GoldBACH-Euler's TheOrem}

Comment \# 72 from [7], titled Variae Observationes Circa Series Infinitas starts in a tantalizing way:

The remarks I have decided to present here generally refer to that kind of series which are absolutely different from the ones usually considered till now.

But in the same way that to date the only series which have been considered are those whose general terms are given or, at least, the laws under which, given a few terms the rest can be found are known, I will here consider mainly those series that have neither a general term as such nor a continuation law but whose nature is determined by other conditions.

Thus, the most astonishing feature of this kind of series would be the possibility of summing them up, as the known methods till now require necessarily the general term or the continuation law without which it seems obvious that 
we cannot find any other means of obtaining their sums. [Our emphasis. Translation of the whole paper electronically available at [8].]

Euler refers to series whose general term is unknown and which, for that reason, had not been considered before. These series are defined by a special property satisfied by their terms, and are otherwise difficult to characterize. Euler continues with his first example: the GET.

I was prompted to these remarks by a special series communicated to me by Cel. GoldBACH[...]

Theorem 1. Consider the following series, indefinitely continued,

$$
\frac{1}{3}+\frac{1}{7}+\frac{1}{8}+\frac{1}{15}+\frac{1}{24}+\frac{1}{26}+\frac{1}{31}+\frac{1}{35}+\cdots
$$

whose denominators, increased by one, are all the numbers which are powers of the integers, either squares or any other higher degree. Thus each term may be expressed by the formula $\frac{1}{m^{n}-1}$ where $m$ and $n$ are integers greater than one.

The sum of this series is 1 .

The proof presented is the following.

Let

$$
x=1+\frac{1}{2}+\frac{1}{3}+\frac{1}{4}+\frac{1}{5}+\frac{1}{6}+\frac{1}{7}+\frac{1}{8}+\frac{1}{9}+\cdots .
$$

Now, as we have

$$
1=\frac{1}{2}+\frac{1}{4}+\frac{1}{8}+\frac{1}{16}+\frac{1}{32}+\cdots,
$$

it will result, subtracting this series from the former

$$
x-1=1+\frac{1}{3}+\frac{1}{5}+\frac{1}{6}+\frac{1}{7}+\frac{1}{9}+\frac{1}{10}+\cdots ;
$$

thus all powers of two, including two itself, disappear from the denominators remaining all the other numbers.

Also, if from that series above we subtract this one

$$
\frac{1}{2}=\frac{1}{3}+\frac{1}{9}+\frac{1}{27}+\frac{1}{81}+\frac{1}{243}+\cdots
$$

there will result

$$
x-1-\frac{1}{2}=1+\frac{1}{5}+\frac{1}{6}+\frac{1}{7}+\frac{1}{10}+\frac{1}{11}+\cdots ;
$$

and subtracting again

$$
\frac{1}{4}=\frac{1}{5}+\frac{1}{25}+\frac{1}{125}+\cdots
$$

it will remain

$$
x-1-\frac{1}{2}-\frac{1}{4}=1+\frac{1}{6}+\frac{1}{7}+\frac{1}{10}+\cdots .
$$


Proceeding similarly deleting all the terms that remain, we finally get

$$
x-1-\frac{1}{2}-\frac{1}{4}-\frac{1}{5}-\frac{1}{6}-\frac{1}{9}-\cdots=1
$$

or

$$
x-1=1+\frac{1}{2}+\frac{1}{4}+\frac{1}{5}+\frac{1}{6}+\frac{1}{9}+\frac{1}{10}+\cdots
$$

whose denominators, increased by one, are all the numbers which are not powers. Consequently, if we subtract this series from the series we have considered at the beginning

$$
x=1+\frac{1}{2}+\frac{1}{3}+\frac{1}{4}+\frac{1}{5}+\frac{1}{6}+\frac{1}{7}+\frac{1}{8}+\cdots ;
$$

we get

$$
1=\frac{1}{3}+\frac{1}{7}+\frac{1}{8}+\frac{1}{15}+\frac{1}{24}+\frac{1}{26}+\cdots,
$$

series whose denominators, increased by one, are all the powers of the integers and whose sum is one. $\quad$ Q. E. D.

In the proof just offered, there are two remarkable facts:

a) The assignation $x=\sum 1 / n$.

b) The procedure consisting of obtaining new series (and consequently, new results) from adding, subtracting and replacing expressions by known series.

What exactly would a modern mathematician find "incorrect" or "not rigorous" in the former proof?

Clearly that would be point a), that is to say, the fact that Goldbach and Euler refer to the harmonic series $\sum 1 / n$ as if it had a real value assigned to it.

If this difficulty is overlooked, point b) may be justified by the rearrangement theorem as the adding and subtracting of series with positive terms is a common procedure when the series involved are convergent.

As we shall see, Euler was well aware of problem a). Since the harmonic series is divergent, to assign a value to it is as absurd as pretending that $x:=1+2+3+4+\cdots$ is a determined quantity. The real motivation for accepting the argument must have been that the procedure used led to results that were correct in the sense that could be verified by more trustworthy finite means. A salient example is Johan Bernoulli's derivation of the sum of the telescoping series (2).

Starting with

$$
H=1+\frac{1}{2}+\frac{1}{3}+\frac{1}{4}+\cdots
$$

and subtracting from it

$$
H-1=\frac{1}{2}+\frac{1}{3}+\frac{1}{4}+\cdots,
$$


Johan Bernoulli obtains

$$
1=\left(1-\frac{1}{2}\right)+\left(\frac{1}{2}-\frac{1}{3}\right)+\left(\frac{1}{3}-\frac{1}{4}\right)+\cdots,
$$

that is to say, the telescoping series:

$$
\frac{1}{1 \cdot 2}+\frac{1}{2 \cdot 3}+\frac{1}{3 \cdot 4}+\cdots=1
$$

Consequently, this procedure seems to work quite well and produce 'valid' results. But there is a drawback: in some instances, a similar manipulation leads to contradiction. This is the case of Jakob Bernoulli's proof of the divergence of the harmonic series. In 1689 spurred on by his brother's argument above, Jakob reversed it and derived the contradiction $H=H-1$ which proved that $H$ could not be a finite quantity.

Let us examine Jakob's argument closely.

2.1. Jakob Bernoulli's proof of the divergence of $\sum 1 / n$. The following is a slightly reworded version - to suit our needs - of Jakob Bernoulli's proof. The original can be found in [18, pp. 316-323] or, more extensively, in [2].

Let

$$
H=1+\frac{1}{2}+\frac{1}{3}+\frac{1}{4}+\frac{1}{5}+\cdots
$$

Subtracting from here the telescoping series (2) we have

$$
H-\left(\frac{1}{1 \cdot 2}+\frac{1}{2 \cdot 3}+\frac{1}{3 \cdot 4}+\frac{1}{4 \cdot 5}+\cdots\right)=\frac{1}{2}+\frac{1}{3}+\frac{1}{4}+\frac{1}{5}+\cdots .
$$

If now from the series above we subtract this one

$$
\frac{1}{2}=\frac{1}{2 \cdot 3}+\frac{1}{3 \cdot 4}+\frac{1}{4 \cdot 5}+\cdots
$$

there will result

$$
H-\left(\frac{1}{1 \cdot 2}+\frac{2}{2 \cdot 3}+\frac{2}{3 \cdot 4}+\frac{2}{4 \cdot 5}+\cdots\right)=\frac{1}{3}+\frac{1}{4}+\frac{1}{5}+\cdots .
$$

Subtracting again

$$
\frac{1}{3}=\frac{1}{4 \cdot 5}+\cdots
$$

it will remain

$$
H-\left(\frac{1}{1 \cdot 2}+\frac{2}{2 \cdot 3}+\frac{3}{3 \cdot 4}+\frac{3}{4 \cdot 5}+\cdots\right)=\frac{1}{4}+\frac{1}{5}+\cdots .
$$

Proceeding similarly all the terms on the right hand side will eventually be deleted, and we shall have

$$
H-\left(\frac{1}{1 \cdot 2}+\frac{2}{2 \cdot 3}+\frac{3}{3 \cdot 4}+\frac{4}{4 \cdot 5}+\cdots\right)=0
$$


or, after simplification,

$$
H=\frac{1}{2}+\frac{1}{3}+\frac{1}{4}+\frac{1}{5}+\cdots
$$

Consequently,

$$
H=H-1
$$

and in Jakob's words, "the whole equals the part" which is impossible for a finite quantity.

We see then how, with similar manipulations to those performed in the proof of the GET, we reach a contradiction. This is caused, quite obviously, by the careless use of divergent series. As late as 1826, Abel echoed this state of affairs saying that:

Divergent series are the invention of the devil, and it is a shame to base on them any demonstration whatsoever. [...] with the exception of the geometrical series, there does not exist in all of mathematics a single infinite series the sum of which has been determined rigorously. [...] That most of these things are correct in spite of that is extraordinarily surprising. [9, p. 170]

Euler knew about the divergence of the harmonic series. He acknowledges it several times in the Variae Observationes and in 1734 he had even written a paper about it [6] in which, among other results, he had obtained:

$$
\lim _{n \rightarrow \infty}\left(1+\frac{1}{2}+\frac{1}{3}+\cdots+\frac{1}{n}-\log n\right)=\gamma
$$

where $\gamma$ is Euler's gamma constant (in fact (3) may be considered $\gamma^{\prime}$ s definition). Later, in his Introductio in Analysin Infinitorum (1748) he provided a completely different argument for the divergence of $\sum 1 / n$ based on the expansion of $\log (1-x)^{-1}$. See [3, pp. 29-31].

Therefore Euler was perfectly conscious of the fragility of the method of deriving new results using divergent series. How come he accepted Goldbach's proof of the GET without any qualm or objection about its validity? Did he not notice the difference between divergent and convergent series or he simply did not care?

This is not the case. As Laugwitz points out in [11, p. 14] and [12] Euler knew the difference very well. In the same paper mentioned above, [6], dedicated to the different harmonic series (series whose general term is $c /(a+n b), n=1,2, \ldots)$, and just before deriving (3) Euler had written:

Although the terms of these series constantly decrease, if indefinitely continued the sum of the series is always infinite. In order to prove this we do not need to find a method for summing these series but the truth will easily come out from the following principle. Series with a finite sum when indefinitely continued, do not increase this sum even if continued 
to the double of its terms. The quantity which is increased behind an infinity of terms actually remains infinitely small. If this were not the case, the sum of the series would not be determined and consequently, would not be finite. As a result of this it follows that if what remains when the terms are continued beyond the place where they begin to become infinitesimal was a finite magnitude, the sum of the series would necessarily be infinite. Therefore from this principle we may judge whether any proposed series has an infinite or a finite sum. [Our translation and our emphasis.]

These words show unmistakably that Euler gave credit to the consideration of different infinitely large and infinitely small numbers. They also hint at the fact that he could be much more explicit and accurate than he usually was in matters regarding convergence had he been interested in doing so. Actually, the emphasized fragment can be clearly considered as a criterium of convergence for series (of positive terms).

The acceptance of Goldbach's proof seems to lie in the fact that, at the time, Euler (and most of his contemporaries) actually manipulated a model of real numbers which included infinitely large and infinitely small numbers. A model that much later Bolzano [11, pp. 19-21] would try to build on solid grounds and that today is called "nonstandard" after A. Robinson definitely established it in the 1960's [16].

\section{A FEW STROKES OF NONSTANDARD ANALYSIS}

For a short and quick introduction to Robinson's nonstandard analysis we recommend Lighstone's excellent paper in this MONTHLY [13]. Here we can only outline some intuitive ideas.

The fundamental notion at the base of nonstandard analysis is the assumption of the existence of an infinitely large positive integer, say $\Omega$, to obtain with its addition an extended number system, much in the same way as the adjunction of $i:=\sqrt{-1}$ to $\mathbb{Z}$ leads to the Gaussian integers, or its adjunction to the real numbers leads to the complex numbers.

In the case of nonstandard we have to postulate a sequence of relations

$$
0<\Omega, 1<\Omega, 2<\Omega, 3<\Omega, 4<\Omega, \ldots
$$

instead of the single one $i:=\sqrt{-1}$, and we must make the fundamental assumption that the laws of arithmetic continue to hold.

This immediately leads to the existence of a legion of new integers as $-\Omega, \Omega-1, \Omega+1, \Omega^{2}, \Omega^{\Omega}$, etc. In fact, the natural ordering must be preserved and we get something like

$$
1,2,3, \ldots ; \ldots, \Omega-1, \Omega, \Omega+1, \ldots, 2 \Omega, \ldots, \Omega^{2} \ldots .
$$

Notice the use of the semicolon to separate the standard natural numbers from the nonstandard ones. 
If we think of $\mathbb{Z} \subset \mathbb{Q} \subset \mathbb{R}$ then we get $\mathbb{Z}(\Omega) \subset \mathbb{Q}(\Omega) \subset \mathbb{R}(\Omega)$, and we end up with the nonstandard real numbers.

In each case the guiding principle in the construction of a model of nonstandard numbers is the principle of permanence of the laws of arithmetic which goes back to Leibniz. In nonstandard analysis jargon it is known as the transfer principle and in our naïve picture may be rather vaguely stated as what is true for finite numbers (i.e. real numbers) is also true for infinite numbers.

The existence of any of such infinitely large numbers, $\omega$, and our commitment to keep the usual operations of arithmetic valid, implies the existence of a number as $\epsilon=1 / \omega$ which verifies $\epsilon<1 / n$ for all $n$. These new numbers, smaller than any positive number, will be called infinitesimals. The existence of infinitesimals implies that any real number $a$ (we now enter the kingdom of real numbers) has a sort of retinue of infinitely close nonstandard numbers, $\{a \pm \epsilon: \forall \epsilon$ infinitesimal $\}$.

There are, though, a few obvious differences between standard and nonstandard numbers. For instance, between two standard natural numbers there is only a finite number of other natural numbers but between two nonstandard natural numbers, there may be an infinity of other nonstandard numbers, e.g. between $\Omega$ and $2 \Omega$ in (5).

In $\mathbb{Q}(\Omega)$ or $\mathbb{R}(\Omega)$ expressions which admit an algebraic closed form like $\sqrt{\Omega}, \sqrt[\Omega]{\Omega}$, etc. acquire a well-defined sense, but in dealing with infinite series the essential point is that they may be viewed as 'ordinary' sums: $\sum_{k=1}^{\omega} a_{k}$.

Thus, given a traditional infinite series, $\sum_{k} a_{k}$ instead of writing $\sum_{k=1}^{\infty} a_{k}$ to denote the limit of the partial sums, we write $\sum_{k=1}^{\omega} a_{k}$ where $\omega$ is an infinitely large number. The question is that now we must talk of different "sums" if different values of $\omega$ are used. If the series $\sum_{k} a_{k}$ is convergent in the standard sense, then any two sums which extend to two different infinitely large indexes are not equal $(=)$ but only infinitesimally close $(\approx)$. If the series is divergent then this difference may be finite or even infinite depending on the indexes considered. For example

$$
1+2+3+4+\cdots+\Omega=\frac{\Omega(\Omega+1)}{2}
$$

is a true identity, though of course each side is an infinitely large number.

With these ideas in mind, Euler's criterium of convergence emphasized in the quotation of Section 2 may be reworded as follows:

Euler's convergence criterium. The series of general term $a_{k}$, $a_{k} \geq 0$ is convergent (has a finite sum) if and only if $\sum_{k=\Omega}^{2 \Omega} a_{k}$ is an infinitesimal for any $\Omega$ infinitely large.

Laugwitz [11, p. 14] rephrases Euler's criterium as 
A series (of real numbers) has a finite (i.e., real) sum iff the values of the sum between infinitely large numbers are infinitesimal.

He goes as far as interpreting Euler's words as equivalent to Cauchy's 1821 convergence criterium.

Remark. With Laugwitz's more general formulation if we consider $\sum_{k=\omega_{1}}^{\omega_{2}} a_{k}$, for any two different infinite numbers, $\omega_{1}$ and $\omega_{2}$ instead of $\sum_{k=\Omega}^{2 \Omega} a_{k}$, Euler's criterium is also valid for series of arbitrary terms.

The application of Euler's criterium to the harmonic series,

$$
\sum_{k=\Omega+1}^{2 \Omega} \frac{1}{k}=\frac{1}{\Omega+1}+\frac{1}{\Omega+2}+\cdots+\frac{1}{2 \Omega}>\frac{\Omega}{2 \Omega}=\frac{1}{2},
$$

proves at once its divergence.

In fact, we can easily go a little further and see that:

$$
\sum_{k=\Omega+1}^{2 \Omega} \frac{1}{k} \approx \log 2
$$

Indeed, the nonstandard version of (3) is

$$
H_{\Omega} \approx \log \Omega+\gamma \quad(\text { Euler writes }=)
$$

and it leads us to

$$
\sum_{k=\Omega+1}^{2 \Omega} \frac{1}{k} \approx \sum_{k=1}^{2 \Omega} \frac{1}{k}-\sum_{k=1}^{\Omega} \frac{1}{k} \approx \log 2 \Omega-\log \Omega \approx \log 2 .
$$

All these considerations are the result of the modern and rigorous point of view offered by nonstandard analysis, but we must concede that they are rather well tuned to Euler's words quoted above!

Unfortunately, we cannot deal more fully with nonstandard analysis but we hope that the few glimpses offered will arouse the reader's interest in getting more acquainted with its methods much in the spirit of André Weil's statement that

[...] our students of mathematics would profit much more from the study of Euler's Introductio in Analysin Infinitorum, rather than of the available modern textbooks. [5, p. xii]

\section{Bernoulli's PROOF RE-EXAMined}

Let us retake the thread of section 2. We saw there Bernoulli's 'proof' of the divergence of the harmonic series and the disturbing observation that the same "style" of proof as that offered by Goldbach and Euler, led to a contradiction. This immediately makes the proof of the GET suspicious of error. 
Let us place ourselves at a modern standpoint. Since we cannot write $H:=\sum 1 / n$, we are forced to consider only the partial sums of the harmonic series:

$$
H_{n}=1+\frac{1}{2}+\frac{1}{3}+\frac{1}{4}+\cdots+\frac{1}{n} .
$$

Now, as we only have a finite number of summands, instead of subtracting the whole telescoping series

$$
1=\frac{1}{1 \cdot 2}+\frac{1}{2 \cdot 3}+\frac{1}{3 \cdot 4}+\cdots
$$

we are almost naturally led to consider the subtraction of

$$
1=\frac{1}{1 \cdot 2}+\frac{1}{2 \cdot 3}+\frac{1}{3 \cdot 4}+\cdots+\frac{1}{(n-1) \cdot n}+\frac{1}{n}
$$

which results in

$$
\begin{aligned}
H_{n}-\left(\frac{1}{1 \cdot 2}+\frac{1}{2 \cdot 3}+\frac{1}{3 \cdot 4}+\cdots+\frac{1}{(n-1) \cdot n}\right) \\
\quad=\frac{1}{2}+\frac{1}{3}+\frac{1}{4}+\cdots+\frac{1}{n}+\frac{1}{n} .
\end{aligned}
$$

Now, if from the sum above we subtract this one

$$
\frac{1}{2}=\frac{1}{2 \cdot 3}+\frac{1}{3 \cdot 4}+\cdots+\frac{1}{(n-1) \cdot n}+\frac{1}{n}
$$

we have

$$
\begin{aligned}
H_{n}-\left(\frac{1}{1 \cdot 2}+\frac{2}{2 \cdot 3}+\frac{2}{3 \cdot 4}+\cdots+\frac{2}{(n-1) \cdot n}\right) & \\
& =\frac{1}{3}+\frac{1}{4}+\cdots+\frac{1}{n}+\frac{2}{n} .
\end{aligned}
$$

The same procedure for $1 / 3$ leads to

$$
\begin{aligned}
& H_{n}-\left(\frac{1}{1 \cdot 2}+\frac{2}{2 \cdot 3}+\frac{3}{3 \cdot 4}+\cdots+\frac{3}{(n-1) \cdot n}\right) \\
&=\frac{1}{4}+\cdots+\frac{1}{n}+\frac{3}{n} .
\end{aligned}
$$

and continuing in the same way we finally get

$$
H_{n}-\left(\frac{1}{1 \cdot 2}+\frac{2}{2 \cdot 3}+\frac{3}{3 \cdot 4}+\cdots+\frac{n-1}{(n-1) \cdot n}\right)=\frac{1}{n}+\frac{n-1}{n}=1
$$

or, after simplification,

$$
H_{n}=\frac{1}{2}+\frac{1}{3}+\frac{1}{4}+\cdots+\frac{1}{n}+1
$$

which is no other than the identity $H_{n}=H_{n}$.

Therefore, even if we let $n \rightarrow \infty$ we never get anything like $H=H-$ 1 , whatever consistent meaning we may attach to $H$. The contradiction has disappeared! 
As long as we think of $n$ as a positive integer, the former is a typical case of the use of perfected later means to make an argument rigorous or, as in this case, to explain an apparent contradiction.

But if we allow infinitely large numbers to enter the scene as first class citizens — as it seems obvious that Euler did - we may consider $n$ an infinite positive integer, $n=\omega$. It makes no difference at all to the proof above and we again reach the tautology $H_{\omega}=H_{\omega}$. Our argument can now be interpreted as the amendment of an error. The crucial point being that any of the $H_{\omega}$ contains all finite unit fractions.

It is clear that the contradiction in Bernoulli's proof arises from the following two reasons:

(a) That there are as many "different" harmonic numbers $H_{n}$ as positive integers $n$. When $n$ is infinitely large any of them can do the job since all finite unit fractions appear in any "infinite harmonic number".

(b) That the remainders of the involved series have not been taken into consideration.

The problem in Jakob Bernoulli's argument came not from the acceptance of infinitely large numbers, but from the use of methods of proof suited only for Weierstrassian mathematics when applied to them, even though Weierstrass was still one hundred and fifty years in the future!

\section{An ELEMENTARY PRoOf of the GET}

The GET appears as problem 132 in the excellent book by Konrad Knopp [10, p. 273]. It is there where we came across it and then proposed it at a Problem Solving Seminar at the Catalan Mathematical Society. As we later discovered, the simple and elegant proof we had found at the Seminar already existed (as almost everything else in mathematics!). It had appeared in this MonThLY [15] in a very synthetic way. We reproduce it for the sake of completeness.

5.1. Solution of the GET by University of South Alabama Problem Group. Let $S$ be the set of positive integers that are powers and $T$ its complement, that is, the nonpowers.

$$
\begin{aligned}
\sum_{s \in S}(s-1)^{-1} & =\sum_{k \geq 2} \sum_{a \in T}\left(a^{k}-1\right)^{-1}=\sum_{k \geq 2} \sum_{a \in T} \sum_{i \geq 1} a^{-i k} \\
& =\sum_{n \geq 2} \sum_{k \geq 2} n^{-k}=\sum_{n \geq 2}(n(n-1))^{-1}=1
\end{aligned}
$$

In order to emphasize the fact that Euler's treatment is more direct and, in a sense, needs less ingenuity than any modern treatment let us examine the ideas that are behind this short proof. 
Remarks on the proof. The proof above is based on the well-known elementary identity

$$
\frac{1}{n-1}=\frac{1}{n}+\frac{1}{n(n-1)}
$$

which will be referred to as the telescoping identity.

Let us assume that the series (1) is convergent and let $A$ be its sum

$$
A=\frac{1}{3}+\frac{1}{7}+\frac{1}{8}+\frac{1}{15}+\frac{1}{24}+\frac{1}{26}+\cdots .
$$

Notice that the terms in this sum are of the form

$$
\frac{1}{a^{m}-1} \text {. }
$$

The difficulty in summing the series (10) arises from the fact that, as Euler points out, we cannot use (11) as its general term. This is because, on the one hand, the series depends on two indexes: the base $a$ and the exponent $m$ and, on the other hand, to the fact that repetitions must be avoided.

Let us concentrate on expression (11), and tentatively let us use on it the telescoping identity (9) with $n=a^{m}$. We obtain

$$
\frac{1}{a^{m}-1}=\frac{1}{a^{m}}+\frac{1}{a^{m}\left(a^{m}-1\right)} \text {. }
$$

We see how (11) splits in two summands of different "shapes": $1 / n$ and $1 /[n(n-1)]$.

If we let $a$ remain fixed and $m$ runs over the integers greater than 1 , then the first summand of

$$
\sum \frac{1}{a^{m}}+\sum \frac{1}{a^{m}\left(a^{m}-1\right)}
$$

becomes a geometric series which can be evaluated in closed form:

$$
\sum_{m \geq 2} \frac{1}{a^{m}}=\frac{1}{a(a-1)} .
$$

This is correct because if the original sum contains all powers, it must contain all powers of each number $a$, and since repetitions are avoided it must contain each power of each number $a$ only once.

After this, quite unexpectedly, both summands in (12) take the same shape. One is

$$
\sum_{a} \frac{1}{a(a-1)}
$$

and the other

$$
\sum_{a} \sum_{m \geq 2} \frac{1}{a^{m}\left(a^{m}-1\right)}=: \sum_{b} \frac{1}{b(b-1)}
$$


where $b$ runs over the integers that are powers. After these elementary transformations,

$$
A=\sum_{a} \frac{1}{a(a-1)}+\sum_{b} \frac{1}{b(b-1)} .
$$

If we only knew that the $a$ 's and the $b$ 's taken together amount to all integers greater than 1 without repetitions, then (14) would precisely be Bernoulli's telescoping series (2) whose sum we know to be 1 .

Is that the case? Can we prove it? A little reflection shows that the answer to both questions is affirmative since a positive integer $n$ is a power if and only if it is a power of a nonpower. That is to say, if it admits an expression $n=a^{m}$ where $a$ is a nonpower and $m \geq 2$. Moreover, in this case and only in this case, $a$ and $m$ are unique. Both statements are a direct consequence of the Fundamental Theorem of Arithmetic.

Following this line of thought the reader should have no difficulty in reconstructing the proof offered above. It is only necessary to reverse the argument, making use of the rearrangement theorem for convergent series of positive terms and of two well-known elementary facts:

(a) The sum of the geometric series (13) which Goldbach and Euler also use in their proof and

(b) The sum of the telescoping series (2) in the last step.

It is interesting to observe that these two results may be derived by iteration of the telescoping identity (9) in two different ways which are totally within Euler's spirit of formal manipulation. This point of view, besides having some class-room value will be useful in the next section.

First, upon substitution of $n-1$ for $n$ in the telescoping identity we get

$$
\frac{1}{n-1}=\frac{1}{(n-1) n}+\frac{1}{n}=\frac{1}{(n-1) n}+\frac{1}{n(n+1)}+\frac{1}{n+1}
$$

and iteration leads to

$$
\frac{1}{n-1}=\frac{1}{(n-1) n}+\frac{1}{n(n+1)}+\cdots+\frac{1}{(n+m-1)(n+m)}+\frac{1}{n+m}
$$

for any positive integer $m$. We may then state:

Lemma 1. For any two positive integers $n, k, 2 \leq n<k$ :

$$
\frac{1}{n-1}=\frac{1}{(n-1) n}+\frac{1}{n(n+1)}+\cdots+\frac{1}{(k-1) k}+\frac{1}{k}
$$

(We have already used Lemma 1 in (7) and (8).)

Second, the same procedure but iterating on $1 /(n-1)$ leads to

$$
\frac{1}{n-1}=\frac{1}{n}+\frac{1}{n} \frac{1}{(n-1)}=\frac{1}{n}+\frac{1}{n^{2}}+\frac{1}{n^{2}(n-1)},
$$

which now gives rise to 
Lemma 2. For any two positive integers $n, n \geq 2$ and $k, k \geq 1$ :

$$
\frac{1}{n-1}=\frac{1}{n}+\frac{1}{n^{2}}+\cdots+\frac{1}{n^{k}}+\frac{1}{n^{k}(n-1)} .
$$

\section{GoldBACH AND EuleR's PROOF REVISITED}

We are finally prepared to reconsider Goldbach and Euler's proof of the GET. We will see how, with almost the same phrasing, the proof can be made rigorous from both points of view, the standard and the nonstandard.

As in the case of Bernoulli's proof, we can either think of $\boldsymbol{n}$ as a finite natural number or as an infinite nonstandard natural number,

$$
H_{\boldsymbol{n}}=1+\frac{1}{2}+\frac{1}{3}+\frac{1}{4}+\frac{1}{5}+\frac{1}{6}+\cdots+\frac{1}{\boldsymbol{n}} \text {. }
$$

Let $k_{2}$ be defined by $2^{k_{2}} \leq \boldsymbol{n}<2^{k_{2}+1}$. Using Lemma 2 , we can write

$$
1=\frac{1}{2}+\frac{1}{2^{2}}+\frac{1}{2^{3}}+\cdots+\frac{1}{2^{k_{2}}}+\frac{1}{2^{k_{2}} \cdot 1},
$$

and subtracting this series from the former (16),

$$
H_{\boldsymbol{n}}-1=1+\frac{1}{3}+\frac{1}{5}+\frac{1}{6}+\frac{1}{7}+\frac{1}{9}+\cdots+\frac{1}{\boldsymbol{n}}-\left(\frac{1}{2^{k_{2}} \cdot 1}\right) .
$$

Hence, all powers of two, including two itself, disappear from the denominators remaining the rest of integers up to $\boldsymbol{n}$.

If now from (17) we subtract

$$
\frac{1}{2}=\frac{1}{3}+\frac{1}{3^{2}}+\frac{1}{3^{3}}+\cdots+\frac{1}{3^{k_{3}}}+\frac{1}{3^{k_{3}} \cdot 2},
$$

obtained again from (15) where $k_{3}$ is defined by $3^{k_{3}} \leq \boldsymbol{n}<3^{k_{3}+1}$, it will result,

$$
H_{\boldsymbol{n}}-1-\frac{1}{2}=1+\frac{1}{5}+\frac{1}{6}+\frac{1}{7}+\frac{1}{10}+\cdots+\frac{1}{\boldsymbol{n}}-\left(\frac{1}{2^{k_{2}} \cdot 1}+\frac{1}{3^{k_{3}} \cdot 2}\right) .
$$

Notice that $k_{2} \geq k_{3} \geq \cdots$. In fact for $m>\sqrt{\boldsymbol{n}}$ we get $k_{m}=1$.

Proceeding similarly we end up by deleting all the terms that remain and we finally get

$$
\begin{aligned}
H_{\boldsymbol{n}}-1-\frac{1}{2}-\frac{1}{4}-\frac{1}{5} & -\frac{1}{6}-\frac{1}{7}-\frac{1}{10}-\cdots-\frac{1}{\boldsymbol{n}} \\
& =1-\left(\frac{1}{2^{k_{2}} \cdot 1}+\frac{1}{3^{k_{3}} \cdot 2}+\cdots+\frac{1}{\boldsymbol{n} \cdot(\boldsymbol{n}-1)}\right) .
\end{aligned}
$$

This last expression has been obtained assuming $\boldsymbol{n}$ is a nonpower. If $\boldsymbol{n}$ is a power, then $1 / \boldsymbol{n}$ will have disappeared at some stage and the last fraction to be removed from (17) will be $1 /(\boldsymbol{n}-1)$ which for $\boldsymbol{n} \neq 9$ will be a nonpower (this is Catalan's conjecture that 8 and 9 are the 
only consecutive powers that exist. The conjecture has recently been proved [14]). The corresponding expression will be:

$$
\begin{aligned}
H_{\boldsymbol{n}}-1-\frac{1}{2}- & \frac{1}{4}-\frac{1}{5}-\frac{1}{6}-\frac{1}{7}-\frac{1}{10}-\cdots-\frac{1}{\boldsymbol{n}-1} \\
& =1-\left(\frac{1}{2^{k_{2}} \cdot 1}+\frac{1}{3^{k_{3}} \cdot 2}+\cdots+\frac{1}{(\boldsymbol{n}-1) \cdot(\boldsymbol{n}-2)}\right) .
\end{aligned}
$$

So we shall have

$$
\begin{aligned}
& H_{\boldsymbol{n}}-1-\frac{1}{2}-\frac{1}{4}-\frac{1}{5}-\frac{1}{6}-\frac{1}{7}-\cdots-\frac{1}{\boldsymbol{n}}\left(\text { or } \frac{1}{\boldsymbol{n}-1}\right) \\
= & 1-\left(\frac{1}{2^{k_{2}} \cdot 1}+\frac{1}{3^{k_{3}} \cdot 2}+\cdots+\frac{1}{\boldsymbol{n} \cdot(\boldsymbol{n}-1)}\right)\left(\text { or } \frac{1}{(\boldsymbol{n}-1)(\boldsymbol{n}-2)}\right) .
\end{aligned}
$$

Consequently, if we subtract this series from (16) we obtain

$$
\begin{aligned}
1-\left(\frac{1}{2^{k_{2}} \cdot 1}+\right. & \left.\frac{1}{3^{k_{3}} \cdot 2}+\cdots+\frac{1}{\boldsymbol{n} \cdot(\boldsymbol{n}-1)}\right)\left(\text { or } \frac{1}{(\boldsymbol{n}-1)(\boldsymbol{n}-2)}\right) \\
& =\frac{1}{3}+\frac{1}{7}+\frac{1}{8}+\frac{1}{15}+\frac{1}{24}+\frac{1}{26}+\cdots+\frac{1}{\boldsymbol{n}-1}\left(\text { or } \frac{1}{\boldsymbol{n}}\right)
\end{aligned}
$$

series that contains in its denominators, increased by one, all the powers of the integers up to $\boldsymbol{n}$. We must now take care of the "remainder".

Since for all $m \geq 1$ by the definition of $k_{m}$ we have $\boldsymbol{n}<m^{k_{m}+1} \leq$ $m^{2 k_{m}}$, it follows that $\sqrt{\boldsymbol{n}}<m^{k_{m}}$ and

$$
\frac{1}{m^{k_{m}} \cdot(m-1)} \leq \frac{1}{\sqrt{\boldsymbol{n}}} \cdot \frac{1}{m-1}
$$

which implies

$$
\frac{1}{2^{k_{2}} \cdot 1}+\frac{1}{3^{k_{3}} \cdot 2}+\cdots+\frac{1}{\boldsymbol{n} \cdot(\boldsymbol{n}-1)} \leq \frac{H_{\boldsymbol{n}-1}}{\sqrt{\boldsymbol{n}}}
$$

or

$$
\frac{1}{2^{k_{2}} \cdot 1}+\frac{1}{3^{k_{3}} \cdot 2}+\cdots+\frac{1}{(\boldsymbol{n}-1) \cdot(\boldsymbol{n}-2)} \leq \frac{H_{\boldsymbol{n}-2}}{\sqrt{\boldsymbol{n}-1}}
$$

If we have chosen to consider $\boldsymbol{n}$ a finite integer, $n$, then we may pass to the limit and use Euler's asymptotic value (3) for $H_{n}$

$$
\lim _{n \rightarrow \infty} \frac{H_{n-1}}{\sqrt{n}}=\lim _{n \rightarrow \infty} \frac{\log (n-1)+\gamma}{\sqrt{n}}=0 .
$$

We are done.

But if we are willing to believe in infinite integers and infinitesimals we do not need to pass to the limit. We use again (3) but now as a nonstandard equality:

$$
\frac{H_{\boldsymbol{n}-1}}{\sqrt{\boldsymbol{n}}} \approx \frac{\log (\boldsymbol{n}-1)+\gamma}{\sqrt{\boldsymbol{n}}} \approx 0
$$


and we finally obtain

$$
\frac{1}{3}+\frac{1}{7}+\frac{1}{8}+\frac{1}{15}+\frac{1}{24}+\frac{1}{26}+\cdots+\frac{1}{n-1} \approx 1 .
$$

In this case, though, what we actually get is a different nonstandard number infinitesimally close to 1 for every infinite $\boldsymbol{n}$ considered.

\section{EPILOG}

Unfortunately not all of Euler's maneuvers with infinite numbers and infinitesimals are so easily amended. This is the case for many of the results in the second part of the Variae Observationes (for all the references to the Variae, see [7]). This part deals mainly with Euler's product formula for the Riemann's zeta function which appears in Theorem 8:

$$
\prod_{p \text { prime }} \frac{1}{1-p^{-s}}=1+\frac{1}{2^{s}}+\frac{1}{3^{s}}+\frac{1}{4^{s}}+\frac{1}{5^{s}}+\frac{1}{6^{s}}+\cdots=\zeta(s),
$$

for $s>1$. Euler only treats the case where $s$ is a positive integer.

The proof of (18) follows the same lines as the proof for the special case $s=1$ which is where the real trouble lies and constitutes Theorem 7:

$$
1+\frac{1}{2}+\frac{1}{3}+\frac{1}{4}+\frac{1}{5}+\frac{1}{6}+\cdots=\prod_{p \text { prime }} \frac{1}{1-p^{-1}} .
$$

Euler clarifies that this common value is infinity. In this particular case keeping track of the remainders is much more difficult (if at all feasible). That may be the reason why Euler is so ambiguous in the first part of the paper when he could have been much more rigorous. He proceeds as follows. From

$$
x=1+\frac{1}{2}+\frac{1}{3}+\frac{1}{4}+\frac{1}{5}+\frac{1}{6}+\cdots
$$

Euler obtains

$$
\frac{1}{2} x=\frac{1}{2}+\frac{1}{4}+\frac{1}{6}+\frac{1}{8}+\cdots
$$

which upon subtraction will provide

$$
\left(1-\frac{1}{2}\right) x=1+\frac{1}{3}+\frac{1}{5}+\frac{1}{7}+\frac{1}{9}+\frac{1}{11}+\cdots,
$$

a series with no even denominators. Multiplying this series by $1 / 3$ and subtracting the result from it we get

$$
\left(1-\frac{1}{2}\right)\left(1-\frac{1}{3}\right) \cdot x=1+\frac{1}{5}+\frac{1}{7}+\frac{1}{11}+\frac{1}{13}+\cdots
$$


and so on until all terms on the right-hand side are deleted. Euler concludes

$$
x \cdot \prod_{p \text { prime }}\left(1-\frac{1}{p}\right)=1
$$

which amounts to (19).

If we now consider (6), corollary 1 to Theorem 7 establishes the 'degree' of infinitude of the harmonic series:

$[\ldots]$ if we denote absolute infinity as $\infty$, then the value of this expression $\left[\prod_{p} \frac{1}{1-p^{-1}}\right]$ is $\log \infty$, which is the minimum among all powers of infinity.

Lastly, taking logarithms in (19) Euler derives Theorem 19, the closing theorem of the Variae, the divergence of the series whose terms are the reciprocals of the primes:

$$
\text { (20) } \begin{aligned}
\frac{1}{2}+\frac{1}{3}+\frac{1}{5} & +\frac{1}{7}+\frac{1}{11}+\cdots \\
& =\log \left(1+\frac{1}{2}+\frac{1}{3}+\frac{1}{4}+\frac{1}{5}+\frac{1}{6}+\cdots\right)=\log \log \infty .
\end{aligned}
$$

Euler's carelessness with the different meanings he attaches to the equality sign is remarkable. As they stand, (19) and (20) are unacceptable. To render them correct it is necessary to change the equality signs by $\sim$ with the usual meaning that the quotient of both sides goes to 1 as $n$ goes to infinity. In fact the strong form of both of these related theorems is due to Mertens in 1874 (see [4, p. 6, footnote]) and may be stated as ( $p$ prime and $\Omega$ any infinitely large integer):

$$
\prod_{p \leq \Omega}\left(1-\frac{1}{p}\right) \cdot H_{\Omega} \approx e^{-\gamma} \quad \text { or } \quad \lim _{n \rightarrow \infty} \prod_{p \leq n}\left(1-\frac{1}{p}\right) \cdot H_{n}=e^{-\gamma}
$$

and

$$
\sum_{p \leq \Omega} \frac{1}{p} \approx \log H_{\Omega}+\gamma+B \quad \text { or } \quad \lim _{n \rightarrow \infty}\left(\sum_{p \leq n} \frac{1}{p}-\log H_{n}\right)=\gamma+B
$$

where $B=\sum_{p}\left(\log \left(1-p^{-1}\right)+p^{-1}\right)$.

As a final comment we want to add that, on occasions, Euler's carelessness took him too far. A striking example is Theorem 18 where he claimed to have proved that $\sum_{n=1}^{\infty} \lambda(n) / n=0$, where $\lambda(n)$ is Liouville's function $\left(\lambda(n)=(-1)^{r(n)}\right.$ where $r(n)$ is the number of prime divisors of $n$ counting repetitions), a result which is true but as deep (and as difficult to prove) as the Prime Number Theorem.

Acknowledgments. We are very grateful to our colleague Angel Gil of Universitat Pompeu Fabra for his careful reading of our manuscript and his many valuable suggestions. We also thank Isabel Tornero for correcting the English. 


\section{REFERENCES}

1. B. Burdick and E. Sandifer, Fooling with an Euler Series, http://vax.wcsu. edu/ Sandifer/E72/Eulerser.htm, 2000.

2. William Dunham, The Bernoullis and the harmonic series, College Math. J. 18 (1987), no. 1, 18-23.

3. - Euler: the master of us all, The Dolciani Mathematical Expositions, vol. 22, Mathematical Association of America, Washington, DC, 1999.

4. Harold M. Edwards, Riemann's zeta function, Academic Press Inc., Boston, 1974.

5. Leonard Euler, An introduction to the analysis of the infinite, vol. 1, SpringerVerlag, New York, 1988, Translated by Jonh D. Blanton.

6. Leonhard Euler, De Progressionibus Harmonicis Observationes, Opera Omnia, I, vol. 14, 1734, pp. 87-100.

7. __ Variae Observationes Circa Series Infinitas, Commentarii Academiae Scientiarum Imperialis Petropolitanae 9 (1744), 160-188, Reprinted in Commentationes Analyticae ad theoriam serierum infinitarum pertinentes (1925) Opera Omnia, ser. I, vol. 14, 216-244.

8. __ Several remarks on infinite series, http://dewey.uab.es/lbibiloni/ euler/EulerObservat.pdf, 2000, Translation of [7] by P. Viader (Senior), P. Viader and L. Bibiloni.

9. Morris Kline, Mathematics. The loss of certainty, Oxford University Press, New York, 1980.

10. Konrad Knopp, Theory and Application of Infinite Series, Dover, New York, 1990, Reprint. Original (English translation) by Blackie, London, 1951.

11. Detlef Laugwitz, Theory of Infinitesimals. An Introducction to Nonstandard Analysis, Accademia Nazionale dei Lincei, Roma, 1980.

12. _ On the Historical Development of Infinitesimal Mathematics. Part I, Amer. Math. Monthly 104 (1997), no. 5, 447-455.

13. A. H. Lightstone, Infinitesimals, Amer. Math. Monthly 79 (1972), 242-251.

14. Tauno Metsnkyl, Catalan's conjecture: another old Diophantine problem solved, Bull. Amer. Math. Soc. (N.S.) 41 (2004), no. 1, 43-57 (electronic).

15. University of South Alabama Problem Group and Z. A. Melzak, Solution of problem E2999, Amer. Math. Monthly 93 (1986), no. 5, 402-403.

16. Abraham Robinson, Non-Standard Analysis, North Holland, Amsterdam, 1966.

17. J. D. Shallit and Karel Zikan, Problem E2999, Amer. Math. Monthly 90 (1983), no. $5,334-335$.

18. D. J. Struik (ed.), A source book in mathematics, 1200-1800, Princeton Paperbacks, Princeton University Press, Princeton, NJ, 1986, Reprint of the 1969 edition.

Lluís Bibiloni (lluis.bibiloni@uab.es). Facultat de Ciències de l'Educació, Universitat Autònoma de Barcelona. 08193 Bellaterra, Barcelona, Spain.

Pelegrí Viader (pelegri.viader@upf.edu).

Jaume Paradís (jaume.paradis@upf.edu). Departament d'Economia i Empresa, Universitat Pompeu Fabra, Ramon Trias Fargas 25-27. 08005 Barcelona, Spain. 